\title{
Humanized mouse models reveal an immunologic classification of idiopathic CD4 lymphocytopenia subtypes
}

\author{
Ainhoa Perez-Diez, ${ }^{1}$ Xiangdong Liu, ${ }^{1}$ Virginia Sheikh, ${ }^{1}$ Gregg Roby, ${ }^{1}$ David F. Stroncek, ${ }^{2}$ \\ and Irini Sereti ${ }^{1}$ \\ ${ }^{1}$ HIV Pathogenesis Section, Laboratory of Immunoregulation, National Institute of Allergy and Infectious Diseases, and \\ ${ }^{2}$ Department of Transfusion Medicine, Clinical Center, NIH, Bethesda, Maryland, USA.
}

\begin{abstract}
Idiopathic CD4 lymphocytopenia (ICL) is a clinically heterogeneous immunodeficiency disorder defined by low numbers of circulating $\mathrm{CD4}^{+} \mathrm{T}$ cells and increased susceptibility to opportunistic infections. CD8 ${ }^{+} \mathrm{T}$ cells, NK, and/or B cells may also be deficient in some patients. To delineate possible pathogenic cellular mechanisms in ICL, we compared immune system development and function in NOD-RAGKO- $\gamma$ CKO (NRG) mice transplanted with hematopoietic stem cells from patients with ICL or healthy controls. CD34+ hematopoietic stem cells from healthy controls and patients with ICL reconstituted NRG mice equally well. In contrast, PBMC transfers into NRG mice identified 2 ICL engraftment phenotypes, reconstituting and nonreconstituting (NR), based on the absence or presence of donor lymphopenia. For patients in the NR group, the distribution of lymphocyte subsets was similar in the peripheral blood of both the patient and the corresponding humanized mice. The NR-ICL group could be further divided into individuals whose $\mathrm{CD}^{+} \mathrm{T}$ cells had defects in proliferation or survival. Thus, ICL cellular pathogenesis might be classified by humanized mouse models into 3 distinct subtypes: (a) T cell extrinsic, (b) T cell intrinsic affecting proliferation, and (c) T cell intrinsic affecting survival. Humanized mouse models of ICL help to delineate etiology and ultimately to guide development of individualized therapeutic strategies.
\end{abstract}

Conflict of interest: The authors have declared that no conflict of interest exists.

Copyright: @ 2019 American Society for Clinical Investigation

Submitted: January 29, 2019

Accepted: June 5, 2019

Published: July 25, 2019.

Reference information: /CI Insight. 2019;4(14):e127802. https://doi. org/10.1172/ji.insight.127802.

\section{Introduction}

Idiopathic CD4 lymphopenia (ICL) is a syndrome characterized by circulating numbers of $\mathrm{CD}^{+} \mathrm{T}$ cells below $300 / \mu \mathrm{L}$ with no other infection, condition, or therapy that could explain the lymphopenia, such as HIV, chemotherapy, or malignancies $(1,2)$. Most ICL cases are detected in adulthood after patients suffer opportunistic, recurrent, or long-lasting infections (mostly fungal or mycobacterial; ref. 3), autoimmunity (4-6), and HPV- or EBV-related neoplasias $(7,8)$ that in some cases can be fatal $(9,10)$. Twenty-five years after the CDC defined ICL, its etiology remains elusive, and there is currently no efficacious treatment, other than the use of antibiotics for prophylaxis or treatment of opportunistic infections (11). Therefore, identifying the etiology/ ies of ICL is important not only to characterize the molecular and cellular mechanisms that can trigger and sustain CD4 lymphopenia in humans but also to direct possible therapeutic interventions in afflicted patients.

The clinical manifestations of ICL are heterogeneous and can include other lymphopenias in CD8 ${ }^{+} \mathrm{T}$ cells, NK, and/or B cells $(3,12)$, suggesting that ICL represents the convergent clinical manifestation of a heterogeneous group of diseases, which should be better defined to guide treatment decisions. For instance, previous in vitro studies by our group and others have shown an overall lower response of $\mathrm{CD}^{+} \mathrm{T}$ cells to IL-7 (13) and T cell receptor (TCR) stimulation $(14,15)$, but with high heterogeneity within the patient population. At the cellular level, multiple causes have been suggested to be responsible for ICL, such as a defect of hematopoietic stem cells (HSCs) to create lymphoid precursors (16), low thymic production (17), defective homeostatic- or antigen-driven proliferation (15) or survival, (18) and differential migration to tissues (19). All these potential defects may be originated by lymphoid cell intrinsic or extrinsic molecular defects. In order to distinguish between these possibilities, we set up 2 humanized mouse models in which either HSCs or mature T cells from patients with ICL were removed from their human environment and placed in an in vivo system that allowed the study of the human lymphoid development and function in different tissues and at different time points after transfer. 
A Bone Marrow (24w)

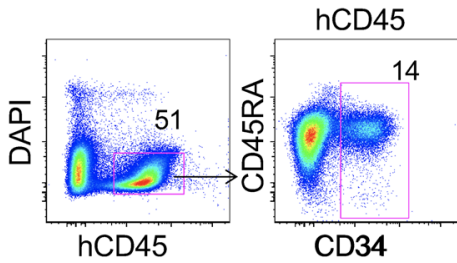

CD34

C Spleen $(24 w)$

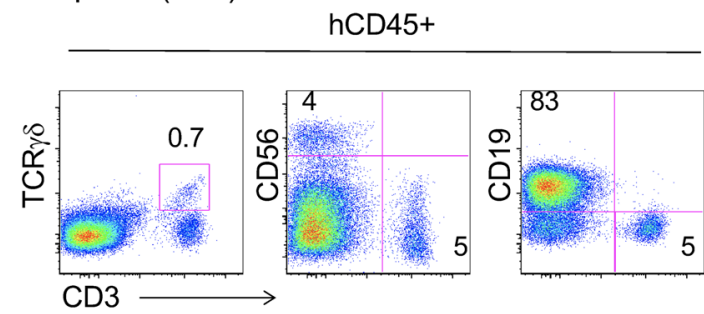

D Spleen (19w)

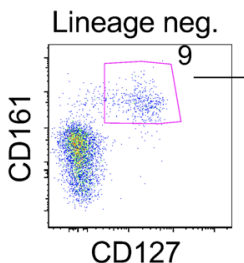

CD127

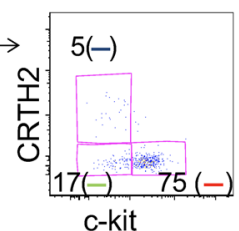

c-kit
B Thymus $(24 w)$

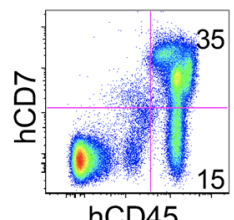

hCD45

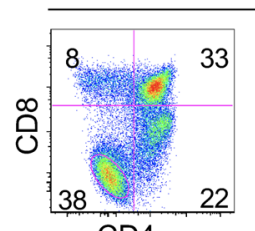

CD4 (a) CD3neg

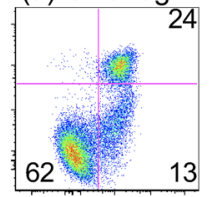

(b) CD3high

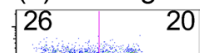

CD3
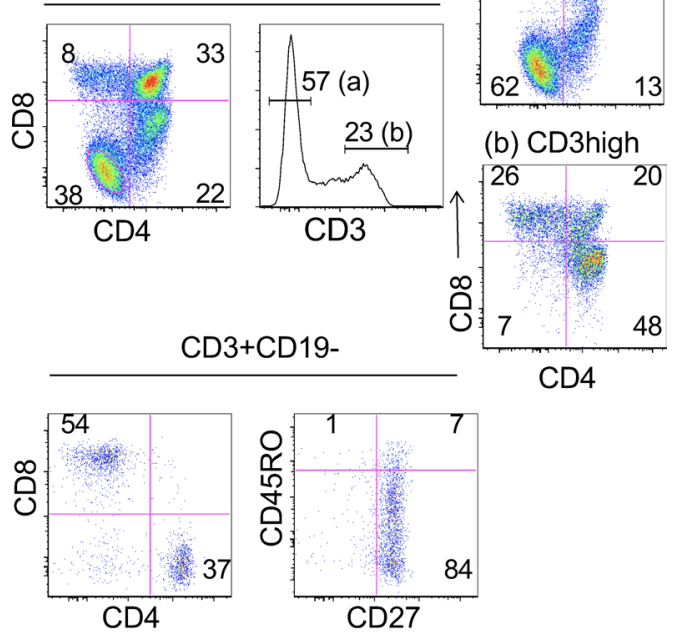

CD4

Figure 1. Characterization of the humanized NRG model using mobilized CD34+ HSCs. Representative phenotype of human CD45 ${ }^{+}$cells in bone marrow (A), thymus (B), and spleen (C), from the same 5-week-old NRG mice 24 weeks after receiving 3.5-Gy irradiation and a 1-million HC hCD34+ cell transfer. (D) Representative phenotype of ILCs (ILC1, ILC2, and ILC3) found in the pooled spleens of 3 hCD34 mice 19 weeks after receiving HC hCD34+. We used the following markers for the linage gate: CD3, CD4, CD19, CD11c, CD14, and CD34. The left panel is gated in lineage-/hCD45 /live cells. Total ILCs are defined as lin-CD127+CD161+ and then ILC1 as c-kit-CRTH2-, ILC2 as c-kit-CRTH2+, and ILC3 as c-kit+'CRTH2- Histograms are overlays of the transcription factor expression for each of the 3 ILC subsets: ILC1 (green), ILC2 (blue), and ILC3 (red). The experiment was done more than 6 times.

The use of immunodeficient strains of mice lacking T and B cells $(20,21)$ and with low NK function (22) as recipients of human PBMCs (hPBMCs) or HSCs started in the late 1980s. In order to diminish rejection of the human cells and improve the level of engraftment, subsequent studies used newly available strains of mice with increasing deficiencies in monocyte (23) and NK functions (24). Most of the studies using humanized mice have focused on infectious diseases, in particular, $\operatorname{HIV}$ infection $(25,26)$, antitumor immune response against primary human tumors (27), transplantation (28), and autoimmune disorders (29), showing their value to understand both disease pathology and effectiveness of potential treatments. Only a few studies, however, have used these models to recapitulate the development of human immune deficiencies using lymphoid cells from monogenic primary immunodeficient patients, when the genetic etiology was already known (30).

In the present study, after comparing 5 different strains of immunodeficient mice, we chose NODRAGKO- $\gamma$ CKO (NRG) mice as recipients of both HSCs and PBMCs to investigate possible etiologies of ICL. We found that ICL HSCs developed and survived in the humanized mice for more than a year equally well as healthy controls (HCs) excluding intrinsic HSC defects. We found, however, that after $\mathrm{PBMC}$ transfer, $\mathrm{CD}^{+} \mathrm{T}$ cells from approximately half of the patients were not able to undergo antigenic and homeostatic expansion to reconstitute the immunodeficient mice. Further analysis showed that the nonreconstituting (NR) ICL CD3 ${ }^{+} \mathrm{T}$ cells could be divided into 2 additional subgroups depending on their inability to either divide or survive under these optimal in vivo conditions.

\section{Results}

Characterization of humanized CD34 $4^{+} N R G$ mice. To test whether patients with ICL have a genetic defect impairing the proper development of $\mathrm{CD}^{+} \mathrm{T}$ cells, we set up a humanized mouse model to compare the capacity of ICL versus HC HSCs to develop in vivo into mature $\mathrm{CD}^{+} \mathrm{T}$ cells. The CD $34^{+} \mathrm{HSCs}$ had min- 
Table 1. Immune and clinical characteristics of patients with ICL and HC donors of CD34+ cells and PBMCs

\begin{tabular}{|c|c|c|c|c|c|c|c|c|c|}
\hline & Donor & $\begin{array}{l}\text { Cells } \\
\text { transferred }\end{array}$ & $\mathrm{CD4}^{+} \mathrm{T}_{\text {cells }}{ }^{\mathrm{A}}$ & CD8 $^{+}$T cells $^{\mathrm{A}}$ & $B$ cells ${ }^{A}$ & NK cells ${ }^{A}$ & Sex & Age (y) & Clinical manifestation \\
\hline \multirow{11}{*}{ Patients } & ICL-18 & $\mathrm{CD}^{4} 4^{+}, \mathrm{PBMCs}$ & 151 & 157 & 45 & 82 & $\mathrm{~F}$ & 58 & None \\
\hline & ICL-24 & $\mathrm{CD}_{4} 4^{+}, \mathrm{PBMCs}$ & 27 & 38 & 98 & 54 & $\mathrm{~F}$ & 59 & $\begin{array}{l}\text { Cryptococcal meningitis, } \\
\text { HPV-related dysplasia }\end{array}$ \\
\hline & ICL-37 & $\mathrm{CD}^{4} 4^{+}, \mathrm{PBMCs}$ & 107 & 83 & 180 & 84 & $M$ & 43 & $\begin{array}{l}\text { Cryptococcal meningitis, herpes } \\
\text { zoster, recalcitrant warts }\end{array}$ \\
\hline & ICL-9 & PBMCs & 183 & 112 & 23 & 106 & $M$ & 67 & Autoimmune phenomena \\
\hline & ICL-26 & PBMCs & 44 & 661 & $211^{B}$ & 155 & $\mathrm{~F}$ & 38 & $\begin{array}{l}\text { Cryptococcal meningitis, } \\
\text { HPV-related dysplasia }\end{array}$ \\
\hline & ICL-28 & PBMCs & 34 & 118 & 434 & 90 & $\mathrm{~F}$ & 37 & $\begin{array}{l}\text { Autoimmune phenomena, } \\
\text { herpes zoster }\end{array}$ \\
\hline & ICL-33 & PBMCs & 70 & 761 & 127 & 102 & $\mathrm{~F}$ & 37 & Recurrent pulmonary infections \\
\hline & ICL-54 & PBMCs & 71 & 732 & 134 & 129 & $\mathrm{~F}$ & 57 & Cryptococcal meningitis \\
\hline & ICL-55 & PBMCs & 291 & 1,732 & $242^{B}$ & 67 & M & 65 & Autoimmune phenomena \\
\hline & ICL-62 & PBMCs & 49 & 214 & $109^{B}$ & 447 & M & 40 & $\begin{array}{l}\text { HPV-related dysplasia, } \\
\text { cryptococcal pneumonia }\end{array}$ \\
\hline & ICL-66 & PBMCs & 15 & 16 & 223 & 140 & $\mathrm{~F}$ & 36 & $\begin{array}{c}\text { Recalcitrant warts, HPV-related } \\
\text { dysplasia }\end{array}$ \\
\hline \multirow[b]{4}{*}{ HCs } & HC 06 & $\mathrm{CD}^{4} 4^{+}$ & 814 & 827 & 407 & 235 & M & 31 & N/A \\
\hline & HC 07 & $\mathrm{CD}^{4} 4^{+}$ & $250^{c}$ & 192 & 84 & 168 & M & 56 & N/A \\
\hline & HC 09 & $\mathrm{CD}^{2} 4^{+}$ & 667 & 562 & 78 & 179 & $M$ & 25 & N/A \\
\hline & HC 12 & $\mathrm{CD} 4^{+}$ & 1546 & 433 & 273 & 420 & $M$ & 52 & N/A \\
\hline
\end{tabular}

${ }^{A}$ Number of cells/ $\mu \mathrm{L}$ of blood at the time of the PBMC transfer into mice for the patients in the ICL group and at the time of bone marrow mobilization for the HCs. ${ }^{B}$ Closest available value to the time of PBMC transfer. ${ }^{C} \mathrm{HC} 07$ included because he had 337 at the time of screening for the mobilization protocol. ${ }^{D}$ Median and range of ages are shown. HC PBMCs were collected from the National Institutes of Health Blood Bank (only age and sex were provided). F, female; M, male; N/A, not available.

imal $\mathrm{CD}^{+} \mathrm{T}$ cell contaminants (Supplemental Figure 1; supplemental material available online with this article; https://doi.org/10.1172/jci.insight.127802DS1), and none of the human CD34+ (hCD34) mice developed graft-versus-host disease (GvHD) during the full length of the experiments, which in some cases reached 57 weeks. We first compared immune-deficient RAGKO- $\gamma \mathrm{CKO}$ mice in 3 different backgrounds (C57BL/6, BALB/c, and NOD) as recipients of HC HSCs, and only the NOD-RAGKO- $\gamma \mathrm{cKO}$ (NRG) mice were consistently and stably engrafted. Age and irradiation dose were optimized (Supplemental Figure 2), and for the remainder of the experiments, we used 3.5-Gy-irradiated 5-week-old NRG mice as hosts of HSCs. Twenty-four weeks after hCD34+ cell transfer, the bone marrow of the hCD34 NRG mice still harbored hCD $45^{+} \mathrm{CD} 34^{+} \mathrm{CD} 45 \mathrm{RA}^{+}$cells (Figure 1A), which contained human $\mathrm{T}$ and $\mathrm{B}$ cell precursors (31). The engraftment was durable over the life span of the mice, as we still found $\mathrm{hCD} 45^{+} \mathrm{CD} 34^{+} \mathrm{CD} 45 \mathrm{RA}^{+}$cells up to 57 weeks after hCD34+ cell transfer (Supplemental Figure 3). Human T cell precursors migrated to the mouse thymus, where they went through $\mathrm{T}$ cell development (Figure 1B). In the periphery, we were able to find not only mature $\mathrm{CD} 4^{+}$and $\mathrm{CD} 8^{+} \mathrm{T}$ cells with mostly naive phenotypes but also NK cells, TCR- $\alpha \beta$ T cells, B lymphocytes (Figure 1C), and even innate lymphoid cells (ILCs) (Figure 1D) with a predominant proportion of ILC3 (c-kit $\left.{ }^{+} / \mathrm{CRTH}^{-}\right)$as previously shown in humanized NOD-SCID- $\gamma \mathrm{cKO}$ (NSG) mice (32). The hCD34 mouse model in NRG mice allows for long-term human bone marrow engraftment and the development of both innate and adaptive human immune systems. 
A PBMC from CD34+ donors B Blood h-CD34 mice ICL donors: 037 O $24 \quad 018$
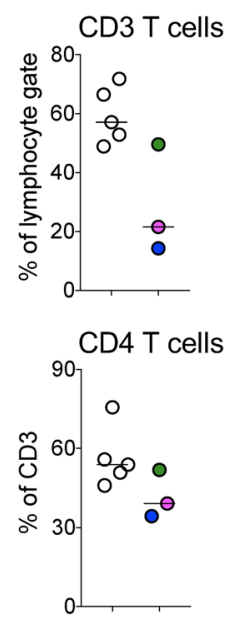

Naive CD4 T cells

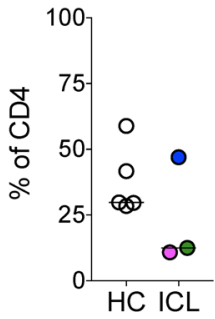

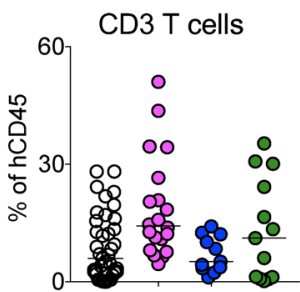

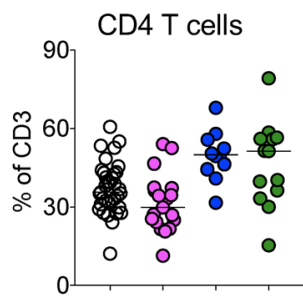

Naive CD4 T cells

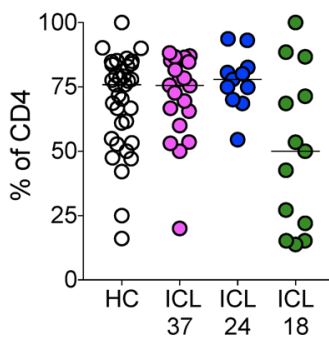

C Spleen $\mathrm{h}-\mathrm{CD} 34$

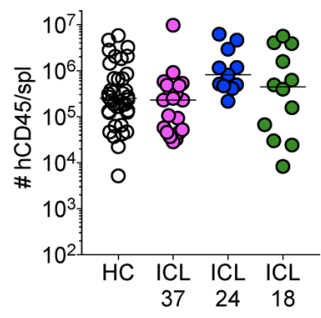

D Spleen h-CD34

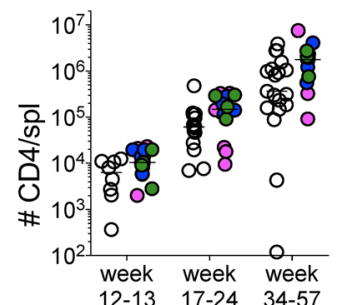

E

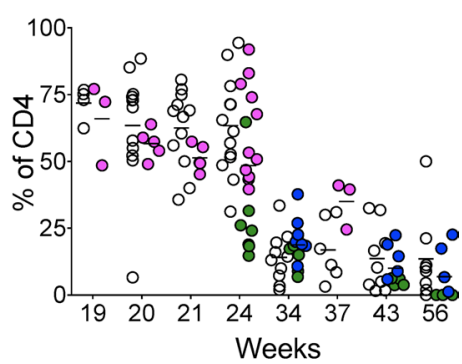

Figure 2. CD34+ cells from patients with ICL and HCs develop similarly in vivo into mature $\mathrm{CD4}{ }^{+} \mathrm{T}$ cells. (A) Percentages of $\mathrm{CD}^{+}, \mathrm{CD}^{+}$, and naive CD4 ${ }^{+}$T cells in PBMCs from $5 \mathrm{HCs}$ (open circles) and 3 ICL donors (ICL-37 in pink, ICL-24 in blue, and ICL-18 in green) whose CD34+ cells were transferred into NRG mice to form hCD34 mice. Naive CD4 ${ }^{+} \mathrm{T}$ cells in $\mathrm{hPBMCs}$ were identified as $\mathrm{CD}^{+} \mathrm{CD} 4{ }^{+} \mathrm{CCR7}{ }^{+} \mathrm{CD} 45 \mathrm{RO}^{-}$. (B) Percentages of the same populations as in $\mathbf{A}$ but found in the blood of hCD34 mice 15 to 17 weeks after receiving CD34+ cells. Naive CD4+ ${ }^{+}$cells in hCD34 mice were identified as $\mathrm{CD}^{+} \mathrm{CD} 4^{+} \mathrm{CD} 27^{+} \mathrm{CD} 45 \mathrm{RO} 0^{-}$. Number of $\mathrm{hCD} 45^{+}$cells (C) and $\mathrm{CD} 4^{+} \mathrm{T}$ cells (D) found in the spleen of hCD34 mice 12 to 57 weeks after CD34 transfer. (E) Percentage of naive CD4+ $T$ cells found in blood of hCD34 mice 12 to 56 weeks after CD34 transfer. (B-E) Each symbol represents a single mouse that received either HC (open symbols) or ICL (closed symbols) CD34+ HSCs. Data in B-E correspond to 4 independent experiments. CD34+ cells from ICL-37 were tested in 3 independent experiments and CD34+ cells from ICL-24 and ICL-18 were tested in 2. Each circle represents an individual measurement and the horizontal line represents the median value.

$C D 34^{+}$cells from patients with ICL and HCs follow similar in vivo development into mature CD4 $4^{+}$cells. We next tested the hypothesis that ICL HSCs might harbor a genetic defect/s that impair their CD4 ${ }^{+} \mathrm{T}$ cell development and/or survival. Figure 2A shows the characteristic PBMC ICL phenotype in the 3 patients who donated HSCs, compared with 5 HC PBMCs. As previously described, PBMCs from patients with ICL had overall lower percentages of $\mathrm{CD}^{+}, \mathrm{CD}^{+}$, and naive $\mathrm{CD} 4^{+} \mathrm{T}$ cells compared with PBMCs from HCs $(3,17)$. We next compared the same populations of cells in mice 15 to 17 weeks after receiving CD34+ cells from the same donors. We found no difference in the percentages of hCD45 $5^{+}$(Supplemental Figure 4), $\mathrm{CD}^{+}, \mathrm{CD}^{+}$, and naive $\mathrm{CD} 4^{+} \mathrm{T}$ cells between mice receiving $\mathrm{HC}$ and ICL HSCs (Figure $2 \mathrm{~B}$ ). Because ICL is mostly detected in adults, to test whether the CD4 lymphopenia would develop later in older mice, we allowed hCD34 NRG mice to age for more than a year while analyzing their CD4 ${ }^{+} \mathrm{T}$ cells in the blood and spleen. Once again, we found comparable numbers of $\mathrm{hCD} 45^{+}$and $\mathrm{CD} 4^{+} \mathrm{T}$ cells between $\mathrm{HCs}$ and ICL hCD34 mice at all time points analyzed (Figure 2, C-E). We noticed that in general, the number of CD4 ${ }^{+}$ $\mathrm{T}$ cells in the spleen increased with time (Figure 2D), while the percentage of naive $\mathrm{CD} 4^{+} \mathrm{T}$ cells decreased after week 24 (Figure 2E) as described in a previous report (33), with no differences between HCs and ICL mice (Figure 2, C-E). We also looked at the thymocytes' cellularity and found no differences between HCs and ICL humanized thymi regarding the number or percentages of $\mathrm{CD} 4^{+}$single-positive thymocytes (Sup- 
A

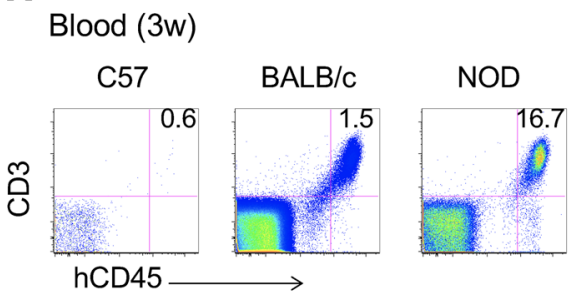

B

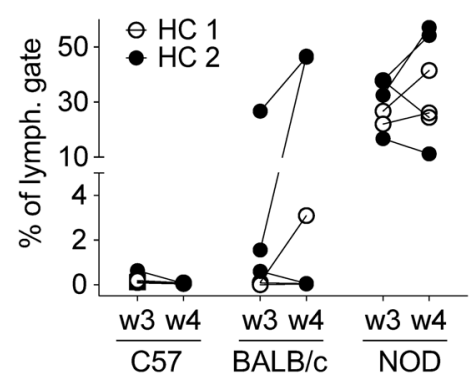

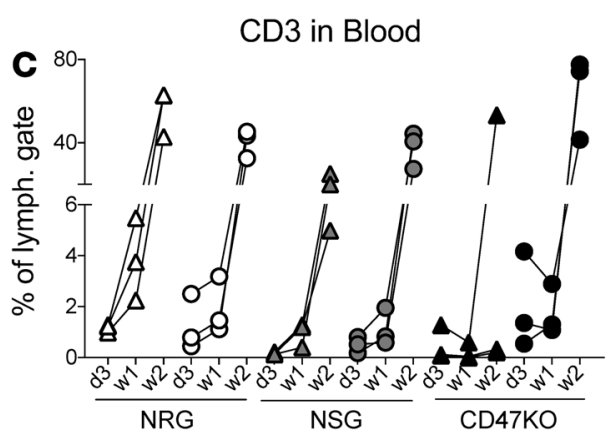

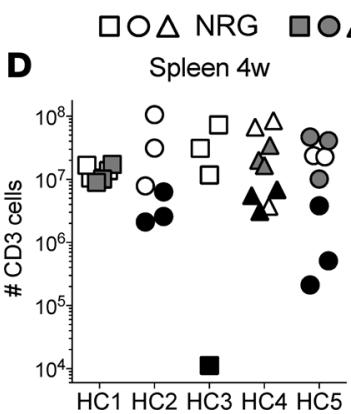

NSG

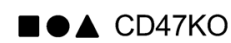

$\mathbf{E}$

Liver $4 \mathrm{w}$

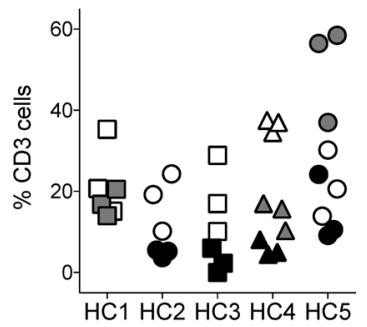

Figure 3. RAGKO- $\gamma$ CKO mice in the NOD background are the optimal hosts for hPBMCs out of $\mathbf{5}$ different strains tested. (A) Dot plot from blood of a representative mouse from either C57BL/6RACKO- $\gamma$ CKO (C57), BALB/CRACKO- $\gamma$ CKO (BALB/C), or NODRACKO- $\gamma C K O$ (NOD) strain 3 weeks after receiving 20 million PBMCs from $2 \mathrm{HC}$ donors. Numbers are the percentage of hCD45+CD3+ within the live lymphocyte gate. (B) Percentage of hCD45+CD3 ${ }^{+}$cells in blood 3 and 4 weeks after PBMC transfer from the same $2 \mathrm{HC}$ donors shown in $\mathbf{A}$. (C) Percentage of hCD45+CD3 ${ }^{+} \mathrm{T}$ cells within the live lymphocyte gate found in blood 3, 7, and 14 days after the transfer of PBMCs from 2 HC donors (donors 4 and 5 in triangles and circles, respectively) into either NRG (open symbols), NSG (gray symbols), or CD47KO (black symbols) mice. (D) Number in spleen and (E) percentage in liver of hCD45+CD3+ cells 28 days after transfer of 5 different $\mathrm{HC}$ PBMC donors into NRG, NSG, or CD47KO mice. (B-E) Each symbol represents an individual mouse. (D and E) Data pooled from 3 independent experiments, with a total of 5 different HC PBMC donors and 3 mice per experiment and per donor. (D and E) Open, gray, and black symbols are NRG, NSG, and CD47KO hosts, respectively.

plemental Figure 5). Altogether, these data show that CD $34^{+}$cells from the 3 tested patients with ICL have no intrinsic defect on in vivo $\mathrm{CD} 4^{+} \mathrm{T}$ cell development, maturation, and long-term maintenance.

$R A G K O-\gamma c K O$ mice in the NOD background are the best hosts for hPBMCS out of 5 different strains tested. We next asked whether mature $\mathrm{CD} 4^{+} \mathrm{T}$ cells from patients with ICL possess a cell-intrinsic defect affecting either their survival, proliferation, and/or migration to tissues. Or if, alternatively, mature ICL CD4 ${ }^{+} \mathrm{T}$ cells once taken away from their patient's environment, are able to follow similar in vivo activation to $\mathrm{HC} \mathrm{CD} 4^{+} \mathrm{T}$ cells. To test this hypothesis, we sought an hPBMC model that would support maximal expansion of transferred adult human T cells with minimal GvHD. Such a model would test both homeostatic- and antigen- driven proliferation capabilities and would allow us to sample not only lymphoid organs but also assess the lymphocyte migration to other tissues. Several strains of immunodeficient mice have been previously shown to support expansion of hPBMCs (34-37) but limited studies compared more than 2 strains side by side. We compared a total of 5 immunodeficient strains of mice as hosts of HC PBMCs to determine which one would be the most suitable for human $\mathrm{CD}^{+} \mathrm{T}$ cells' expansion/survival. First, we tested RAGKO- $\gamma \mathrm{CKO}$ mice in 3 different backgrounds (C57BL/6, BALB/c, and NOD) and found that 3 weeks after hPBMC transfer, the majority of $\mathrm{hCD} 45^{+}$cells in the mouse peripheral blood were $\mathrm{CD}^{+} \mathrm{T}$ cells, albeit they were almost nonexistent in C57 mice (Figure 3A). Both BALB/c and NOD backgrounds supported human T cell survival and expansion, although NOD mice were consistently better based on the percentages of $\mathrm{CD}^{+}$ T cells found in blood (Figure 3, A and B) and spleen (Supplemental Figure 6A). Four weeks after transfer, almost all of the $\mathrm{CD}^{+} \mathrm{T}$ cells displayed an activated phenotype $\left(\mathrm{CD} 45 \mathrm{RO}^{+}\right)$in both $\mathrm{BALB} / \mathrm{c}$ and NOD mice (Supplemental Figure 6B), with the former showing more severe GvHD than the latter (Supplemental Figure 6C). We next compared NRG versus both NSG and C57BL/6-RAGKO- $\gamma$ cKO-CD47KO (CD47KO) mice as recipients of 5 different $\mathrm{HC}$ PBMCs and looked at $\mathrm{CD}^{+} \mathrm{T}$ cell engraftment at different time points. NRG mice reconstituted slightly faster than the other 2 strains (Figure 3C). PBMCs in both NRG and NSG 
A
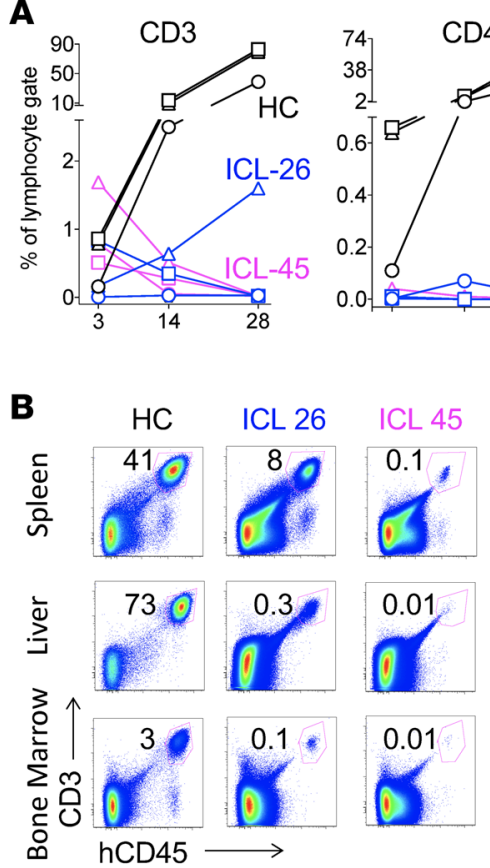

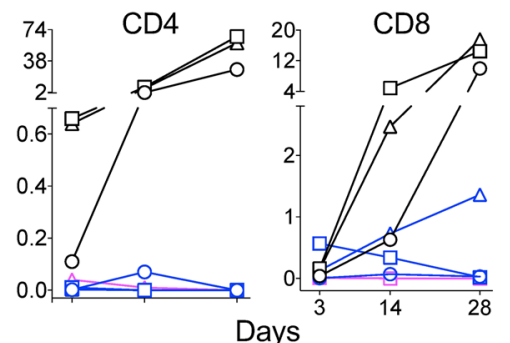

Days

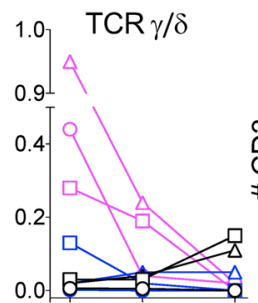

cells transferred

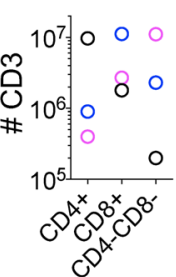

co

(1)
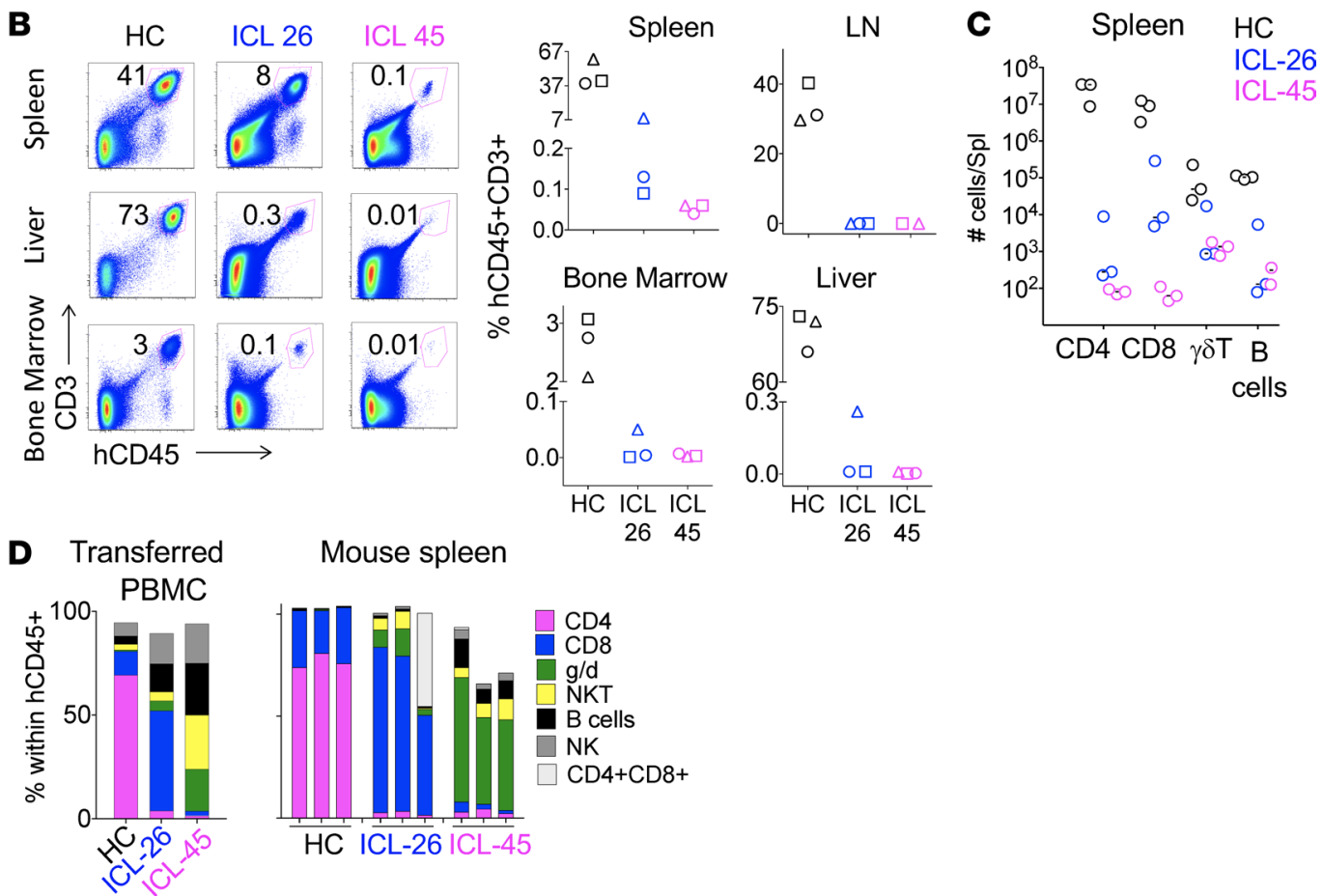

Figure 4. ICL is phenocopied in NRG mice receiving ICL PBMCs. (A) Percentage of $C D 3^{+}, \mathrm{CD}^{+}$, $C D 8^{+}$, and $\mathrm{TCR}-\gamma \delta^{+}$ T cells in blood of hPBMC mice at days 3, 14, and 28 after transfer of PBMCs from HC (black) or patients ICL-26 (blue) or ICL-45 (pink). All percentages calculated from the live lymphocyte gate. Insert represents the number of $\mathrm{CD}^{+}, \mathrm{CD}^{+}$, and $\mathrm{CD}^{+} \mathrm{CD}^{-} \mathrm{CD} 8^{-}$cells (where $\gamma \delta \mathrm{T}$ cells are included) that each experimental group of mice received. (B) Representative dot plots (left) and compiled data (right), showing the percentage of hCD45+CD3 ${ }^{+}$cells found in spleen, LN, bone marrow, and liver, 4 weeks after PBMC transfer, gated on live events. (C) Numbers of CD4 ${ }^{+}, \mathrm{CD}^{+}$, $\gamma \delta \mathrm{T}$ cells, and B cells found in spleens of hPBMC mice 4 weeks after PBMC transfer. (D) Percentages of the different populations found within the live hCD45+ gate in either the original PBMC inoculum (left) or in the spleen of individual mice 4 weeks after PBMC transfer (right). When the total percentage does not add up to 100, it is due to a subset of events that were hCD45+ but negative for any of the markers used. (A-D) Data from the same experiment; the same symbols represent the same individual mice.

mice reconstituted better than in $\mathrm{CD} 47 \mathrm{KO}$ mice when looking at $\mathrm{CD}^{+} \mathrm{T}$ cells in the spleen (Figure 3D and Supplemental Figure 6D). The development of GvHD was more dependent on donor PBMCs than on the host strain and did not always correlate with higher $\mathrm{T}$ cell expansion or tissue infiltration (Supplemental Figure 6E). NRG mice were selected as hosts for future experiments based on their most reliable support of human $\mathrm{CD}^{+} \mathrm{T}$ cell expansion and survival with relatively low levels of GvHD.

Patients with ICL can be phenocopied in NRG mice receiving ICL PBMCs. We next transferred PBMCs from either HCs or patients with ICL into NRG mice and followed their T cell populations at different time points and in different organs to assess their reconstitution capacity. Mice receiving ICL PBMCs had a lower percentage of $\mathrm{CD}^{+} \mathrm{T}$ cells in blood by day 14 compared with mice receiving HC PBMCs (Figure 4A). Interestingly, the lymphopenia observed in ICL hPBMC mice was not restricted to the $\mathrm{CD} 4^{+} \mathrm{T}$ cell compartment, as $\mathrm{CD}^{+}$and $\gamma \delta \mathrm{T}$ cells were also found at much lower levels than in HC hPBMC mice (Figure 4A). This finding was despite the fact that ICL $\mathrm{hPBMC}$ mice received higher numbers of both $\mathrm{CD} 8^{+}$and $\gamma \delta \mathrm{T}$ cells than 
A

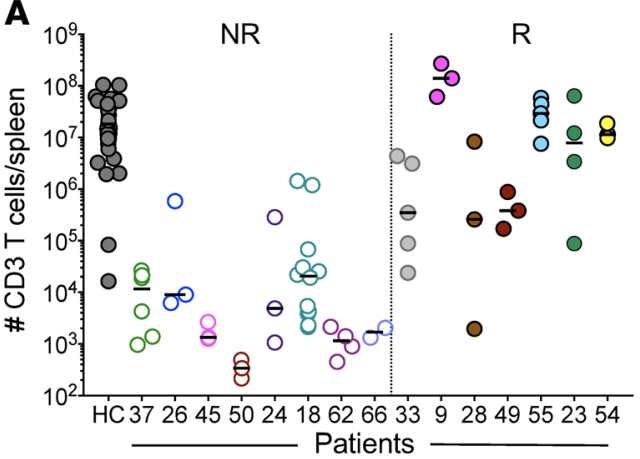

B Naive CD3 T

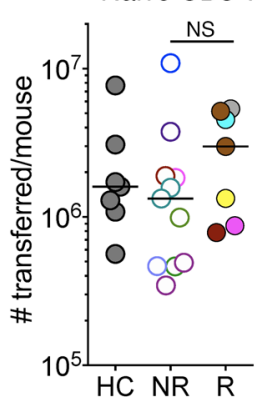

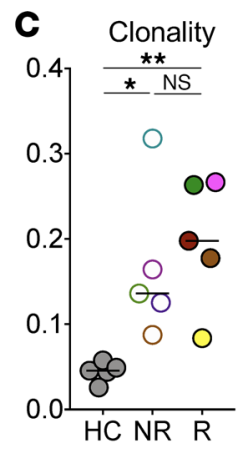

D

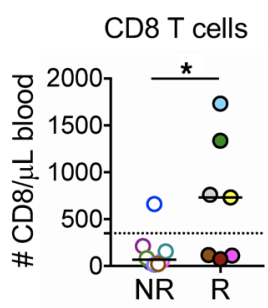

E

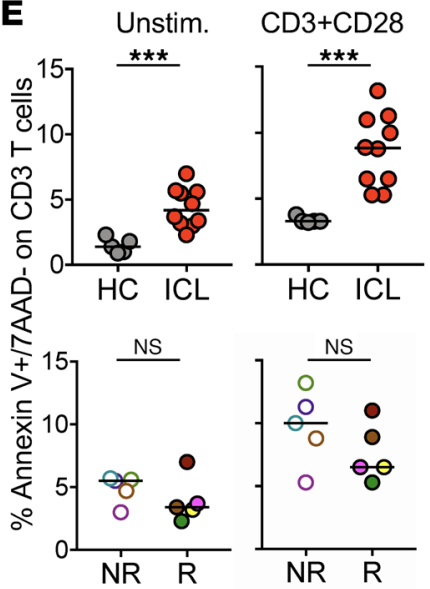

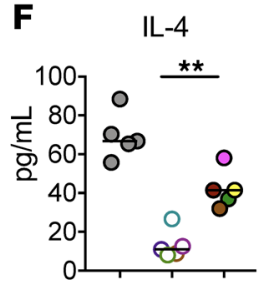
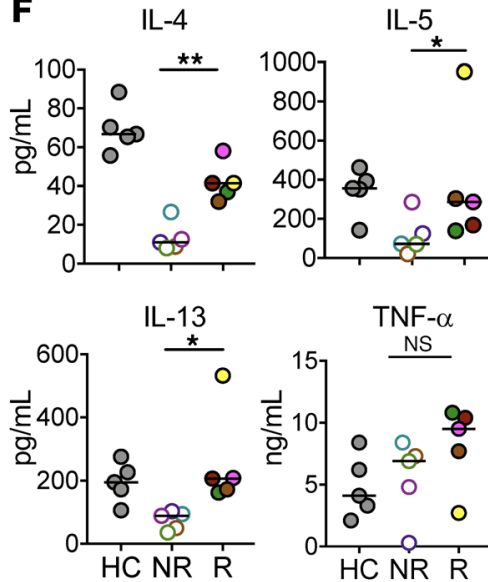

Figure 5. The hPBMC model identifies 2 subclasses of patients in the ICL group depending on their PBMC reconstitution capability in NRG mice. (A) Number of $\mathrm{CD}^{+} \mathrm{T}$ cells found in the spleen of hPBMC mice 4 weeks after transfer of either HCs or ICL PBMCs. Each symbol represents an individual mouse. (B) Number of naive CD3 ${ }^{+} \mathrm{T}$ cells injected per mouse for each of the donors shown in $\mathbf{A}$. Each symbol represents 1 donor. In $\mathbf{A}$ and $\mathbf{B}$, pooled data from 8 independent experiments conducted using 8 different $\mathrm{HC}$ donors and 15 different patients with ICL. (C) Clonality of the CD $3^{+} \mathrm{T}$ cells from 5 donors per group (HC, NR, and R). (D) Number of $C D 8^{+} T$ cells per microliter of blood in patients with ICL at the time of mouse transfer. Dotted line represents the number of $C D 8^{+} \mathrm{T}$ cells $(350$ cells $/ \mu \mathrm{L}$ ) below which the patient is considered CD8 lymphopenic. (E) Percentage of apoptotic CD3 ${ }^{+} T$ cells (defined as annexin $V^{+} 7-A A D^{-}$) after 24 hours of in vitro culture with either media (Unstim.) or CD3 plus CD28 stimulation. (F) Cytokines measured in the supernatant after $C D 3$ plus $C D 28$ stimulation in same experiment as $\mathbf{E}$. Each symbol represents the average measured cytokine level from duplicate wells. Throughout the panels, the same symbol color represents the same patient. ns, not significant; ${ }^{*} P<0.05 ;{ }^{* *} P<0.005 ;{ }^{* *} P<0.001$

HC hPBMC mice (Figure 4A, insert), and that by day 3, ICL hPBMC mice still showed $\mathrm{CD} 8^{+}$and $\gamma \delta \mathrm{T}$ cell percentages similar and higher, respectively, than HC hPBMC mice (Figure 4A). Similar to blood, the spleen, lymph nodes (LNs), bone marrow, and liver from ICL hPBMC mice showed much lower percentages of $\mathrm{CD}^{+} \mathrm{T}$ cells than HC hPBMC mice (Figure 4B). Therefore, and similar to our previous findings in patients with ICL (38), the lymphopenia observed in ICL hPBMC mice was not caused by increased migration and/ or retention of ICL CD3 ${ }^{+} \mathrm{T}$ cells into lymphoid or nonlymphoid tissues. The numbers of $\mathrm{CD}^{+}, \mathrm{CD}^{+}, \gamma \delta$ $\mathrm{T}$ cells, and $\mathrm{B}$ cells in the spleen corroborated the generalized lymphopenia observed in ICL mice relative to $\mathrm{HC}$ mice (Figure $4 \mathrm{C}$ ). The relative proportions of $\mathrm{CD}^{+}, \mathrm{CD}^{+}, \gamma \delta$, NKT, B cells, and NK cell subsets within the $\mathrm{hCD} 45^{+}$cells found in the spleen were very similar to the proportions in the initial donor (Figure 4D). For instance, spleen from hPBMC mice of ICL-26 had the highest percentage of CD8 ${ }^{+} \mathrm{T}$ cells, analogous to the same patient's PBMCs, and spleen from hPBMC mice of ICL-45 had an overrepresentation of $\gamma \delta \mathrm{T}$ cells, which were also at a higher percentage in the same patient's PBMCs (Figure 4D). Thus, the hPBMC model uncovered a cell-intrinsic inability of mature ICL $\mathrm{CD}^{+} \mathrm{T}$ cells to follow homeostatic- and antigen-driven proliferation and/or survival, which translated in their failure to reconstitute the immune-deficient mice.

The hPBMC model identifies 2 groups of patients with ICL based on their CD $3^{+} T$ cell reconstitution capabilities. We next tested 13 more patients with ICL using the hPBMC model and found that the patients could be divided into 2 major groups depending on the reconstitution achieved by their $\mathrm{CD}^{+} \mathrm{T}$ cells (Figure $5 \mathrm{~A}$ ). 
A

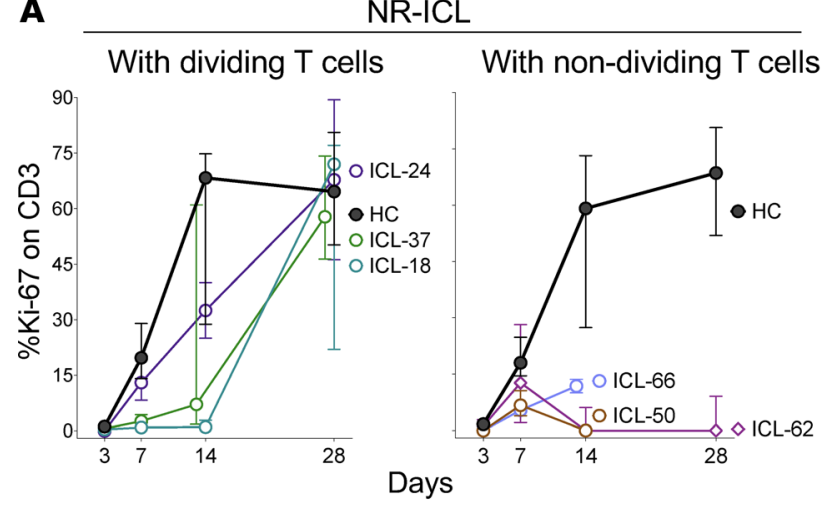

R-ICL

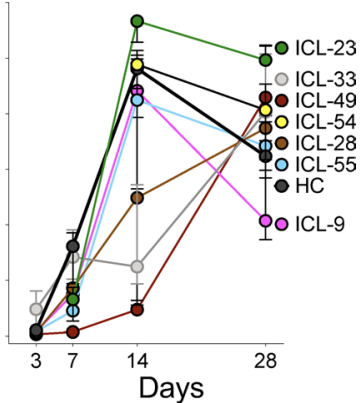

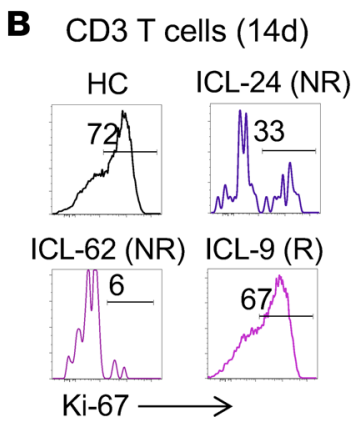

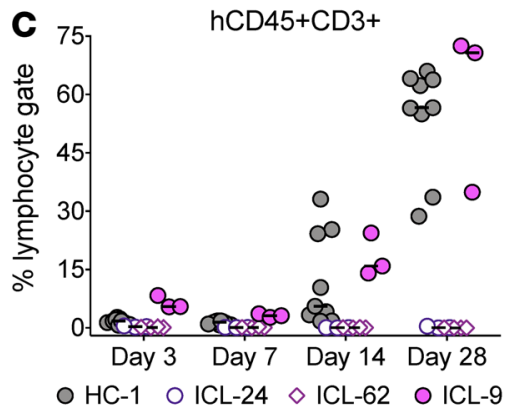

Figure 6. Patients in the NR-ICL group can be further classified based on their T cell capacity to enter cell division in the hPBMC model. (A) Percentages of Ki-67+ cells within the CD3+ ${ }^{+}$cells in blood of hPBMC mice 3, 7, 14, and 28 days after PBMC transfer. ICL-50 and ICL- 66 in the middle panel do not have data at day 28 because none of the mice in those groups had detectable CD3 ${ }^{+} \mathrm{T}$ cells in blood at that time point. Data pooled from 7 out of the 8 independent experiments are shown in Figure $5 \mathrm{~A}$ from which $\mathrm{Ki}-67$ analysis was available. Symbols represent the median and interquartile range from groups with variable number of individual mice ranging between 2 and 17. (B) Histogram of $\mathrm{Ki}-67$ staining on $\mathrm{CD}^{+} \mathrm{T}$ cells at day 14 belonging to 1 mouse representing an $\mathrm{HC}$ and each of the 3 panels shown in $\mathbf{A}$. (C) Percentage of hCD45+CD3+ $\mathrm{T}$ cells in blood of hPBMC mice $3,7,14$, and 28 days after transfer of PBMCs from HCs, or patients ICL-24, ICL-62, or ICL-9. Percentages calculated from the live lymphocyte gate show data from individual mice with the median for each group at each time point. Data are pooled from 2 independent experiments.

Overall, the $\mathrm{CD}^{+} \mathrm{T}$ cells from 8 out of 15 patients were unable to reconstitute the immunodeficient mice (Figure 5A, open circles). The $\mathrm{CD}^{+} \mathrm{T}$ cells from the other 7 patients reconstituted the mice at similar (ICL 9, 55, 23, and 54) or somewhat lower levels (ICL 33, 28, and 49) compared with $\mathrm{HC} \mathrm{CD}^{+} \mathrm{T}$ cells (Figure 5A, closed circles). We named these 2 ICL groups nonreconstituting (NR) and reconstituting (R), respectively. Similar results were obtained when specifically looking at $\mathrm{CD} 4^{+}$or $\mathrm{CD} 8^{+} \mathrm{T}$ cell reconstitution (Supplemental Figure 7, A and B). The difference in reconstitution could not be explained by differences in the initial number of $\mathrm{CD}^{+} \mathrm{T}$ cells transferred, as mice received similar ranges of $\mathrm{CD}^{+} \mathrm{T}$ cells from either HCs or patients with ICL (Supplemental Figure 7C). Patients with ICL have, as a whole, lower percentages of naive $\mathrm{CD}^{+} \mathrm{T}$ cells than $\mathrm{HCs}(3,17)$. To rule out the possibility that lower numbers of naive $\mathrm{CD}^{+} 3 \mathrm{~T}$ cells in the initial transfer, and not cell intrinsic factors, might account for the lack of reconstitution capability by the patients in the NR-ICL group, we calculated the number of naive $\mathrm{CD}^{+} \mathrm{T}$ cells $\left(\mathrm{CD}^{+} \mathrm{CD} 27^{+} \mathrm{CD} 45 \mathrm{RO}^{-}\right)$ transferred per mouse. Figure 5B shows that the number of naive $\mathrm{CD}^{+} \mathrm{T}$ cells transferred did not correlate with their reconstitution capability. The medications that the patients in the ICL group were taking at the time of the PMBC sampling were fairly similar and could not explain the discrepancy between the NR and $\mathrm{R}$ behavior of the ICL $\mathrm{CD} 3^{+} \mathrm{T}$ cells (Supplemental Table 2). While the frequency of the different lymphocyte populations stayed similar to the original inoculum in patients in the NR-ICL group (Supplemental Figure 7, ICL 66), in patients in the R-ICL group, the lymphocyte frequencies reverted or stayed similar to those found in HC hPBMC mice (Supplemental Figure 8, ICL 28 and 9, respectively).

A previous study showed the TCR repertoires from 3 patients with ICL to be highly restricted (39). To see whether a potentially more oligoclonal TCR repertoire in the NR-ICL CD3 ${ }^{+} \mathrm{T}$ cells might explain their lack of xenoantigen recognition, limiting their reconstituting capability in the hPBMC model, we 
sequenced the TCR- $\beta$ variable region from $5 \mathrm{HC}$, NR-ICL, and R-ICL donors from the same time point as their PBMC sampling for the hPBMC mouse model. As described by Signorini et al. (39), patients with ICL showed higher clonality than HCs (Figure 5C); however, no differences were observed between the patients in the NR and R-ICL groups. In contrast, the number of circulating CD8 $8^{+} \mathrm{T}$ cells at the time of PBMC sampling seemed to associate more with the reconstituting capability of the ICL $\mathrm{CD} 3^{+} \mathrm{T}$ cells, as patients with higher $\mathrm{CD} 8^{+} \mathrm{T}$ cell counts were more likely to reconstitute the mice (Figure 5D).

Increased apoptosis of $\mathrm{CD}^{+} \mathrm{T}$ cells has been previously described in a group of 7 patients with ICL (18). We next tested if the inability to reconstitute the NRG mice of some patients with ICL might be explained by an increase in apoptosis of their $\mathrm{CD}^{+} \mathrm{T}$ cells under stimulatory conditions. Figure $5 \mathrm{E}$ shows increased apoptosis in ICL CD3 ${ }^{+} \mathrm{T}$ cells when compared with HC cells in both unstimulated and stimulating conditions after overnight culture. There was no significant difference in apoptosis, however, between the patients in the NR and R-ICL groups. Similar results were obtained when measuring the total number of dead cells and even when the $\mathrm{CD}^{+} \mathrm{T}$ cells were stimulated with splenocytes from NRG mice (Supplemental Figure 9). We also measured T helper cytokine production under the same in vitro conditions. Out of the 13 different cytokines measured and described in the Methods section, only 3 were significantly different between patients in the NR and R-ICL groups, all Th2 cytokines (IL-4, IL-5, and IL-13), and were produced in lower amounts by the NR-ICL CD3 ${ }^{+} \mathrm{T}$ cells than by the R-ICL CD3 ${ }^{+} \mathrm{T}$ cells after TCR stimulation (Figure $5 \mathrm{~F}$ ). Other cytokines such as TNF- $\alpha$ were produced at similar levels between the NR and R groups (Figure $5 \mathrm{~F}$ ), ruling out a possible defect on TCR signaling in the NR-ICL CD3 ${ }^{+} \mathrm{T}$ cells.

Altogether, our data suggest that in the R-ICL patient group, the lymphopenia might be caused by an extrinsic factor that can be reversed once the cells are removed from their patient's environment. While in patients in the NR-ICL group, the lymphopenia might be caused by a cell intrinsic factor for which lower Th2 cytokine production might be a good marker.

Patients in the NR-ICL group can be further classified based on their T cell capacity to enter cell division in the $h P B M C$ model. To test whether a deficiency to enter cell division was responsible for the lack of reconstitution shown by the T cells from patients in the NR-ICL group, we analyzed Ki-67 staining on $\mathrm{CD}^{+} \mathrm{T}$ cells at different time points after transfer. We found that the NR-ICL group could be divided in 2 subgroups depending on whether or not their $\mathrm{T}$ cells were able to enter the cell cycle (Figure 6A), while $\mathrm{CD}^{+} \mathrm{T}$ cells from the R-ICL group divided profusely (Figure 6A). Figure 6B shows an example of Ki-67 staining on $\mathrm{CD}^{+} \mathrm{T}$ cells from 1 representative patient per group. The fact that there are 3 patients with $\mathrm{CD}^{+} \mathrm{T}$ cells capable of entering the cell cycle but unable to reconstitute the mice suggests a higher cell death rate by their $\mathrm{CD}^{+} \mathrm{T}$ cells. For instance, ICL-24 CD3 ${ }^{+} \mathrm{T}$ cells, despite going through in vivo cell division (Figure $6 \mathrm{~A}$ ), failed to reconstitute NRG mice (Figure 5A and Figure 6C). Supporting this possibility is the fact that these 3 patients (ICL-18, ICL-24, and ICL-37) showed the highest percentage of apoptosis from the NR-ICL group after in vitro TCR stimulation (Figure 5E). The hPBMC model, therefore, was able to further identify 2 subgroups within the NR-ICL group. The first subgroup, with T cells entering the cell cycle, identifies patients whose $\mathrm{CD}^{+} \mathrm{T}$ cells might have a specific problem with cell survival. The second group identifies patients whose $\mathrm{T}$ cells are unable to enter the cell cycle in this lymphopenic environment.

\section{Discussion}

Understanding the etiology of ICL has been hindered by the diversity of clinical and immunological phenotypes, suggesting it may represent the end result of different diseases sharing peripheral CD4 lymphocytopenia as their main common feature. In this study, we used an approach that allowed us to test the in vivo behavior of ICL cells in humanized mice. The 2 mouse models described here, with transfers of either HSCs or PBMCs from patients with ICL, allowed us to address basic cellular questions regarding $\mathrm{T}$ cell precursor availability, T cell development, migration, division, and survival. Our data demonstrated that HSCs from the patients with ICL tested were as fit as HSCs from HCs. In contrast, transfer of mature $\mathrm{CD}^{+} \mathrm{T}$ cells unveiled 3 newly identified subgroups of patients with ICL, based on their T cell homeostatic- and antigen-driven expansion and survival when compared with HC T cells.

The aim of this study was to systematically evaluate whether the etiology of ICL is related to cell intrinsic or extrinsic defects at either the HSC level or at the mature T cell level. In contrast with previous work showing lower in vitro clonogenic capability of bone marrow from 5 patients with ICL (16) and lack of mouse immune reconstitution by an ICL patient's CD $34^{+}$cells (40), we found no HSC cell intrinsic problems in the few patients with ICL tested. T cell development could still be potentially impaired in ICL by a failure on any of the criti- 
cal signals that HSCs and T cell precursors require from bone marrow and thymic stroma. The robustness of our model supports the continuation of testing more patients with ICL, particularly when their whole-exome sequencing analysis is available and suggestive of specific de novo genetic defects that could be responsible for the ICL phenotype. In support of this finding, previous work has shown that humanized mice transplanted with HSCs from a patient with an SNP in the forkhead box P3 (FOXP3) gene causing immune dysregulation, polyendocrinopathy, enteropathy, and X-linked syndrome, experienced similar immune dysregulation as patients with this monogenic immune disorder (30). Notwithstanding, by whole-exome sequence analysis, we found that 2 of the 3 patients with ICL tested here had potentially damaging SNPs in protein kinase, DNA-activated, catalytic subunit (PRKDC) and zeta chain of TCR-associated protein kinase 70 genes (ZAP70) (patient ICL-24) and in unc-119 lipid-binding chaperone (UNC119) and Ras-related protein 1 genes (RAP1) (patient ICL-37). Mutations in PRKDC, ZAP70, and UNC119 have been associated with immunodeficiencies 26, 48 and 13, respectively (41-43). Based on the normal development of HSCs from these patients in the hCD34 model, however, a critical role of these SNPs in human lymphocyte development and homeostasis is doubtful. It is possible, however, that these SNPs might play a role in antigen- and homeostatic-driven expansion of mature $\mathrm{T}$ cells because the $\mathrm{CD}^{+}$ T cells from these 2 patients failed to reconstitute in the hPBMC mouse model.

An additional value of the hCD34 model would be as a screening method of candidate mutations by introducing them in HC HSCs before following T cell development in hCD34 mice, which could be more efficient and inexpensive than developing the whole mutant mouse by CRISPR-Cas9 technology (44). Lastly, the hCD34 mouse model could provide a useful tool as a preclinical model to test potential future therapies. In this regard, CRISPR/Cas9-based gene editing has already been successfully employed on human HSCs to correct X-linked severe combined immunodeficiency (SCID)-X1, and it has been tested successfully on the hCD34 mouse model (45).

The hPBMC mouse model allowed us to identify 2 major groups of patients in the ICL group: reconstituting $(\mathrm{R})$ and nonreconstituting (NR), implying classification of ICL groups with cell-extrinsic or -intrinsic etiology, respectively, in regard to patients' adult $\mathrm{CD}^{+} \mathrm{T}$ cells. The R-ICL group might reveal patients whose CD4 lymphopenia might be explained by environmental abnormalities such as microbiome, infection history, and perhaps in combination with yet-unknown genetic predisposing factors. In the NR-ICL patient group, the potential intrinsic factor causing the failure of $\mathrm{T}$ cells to reconstitute the mice was not unique to $\mathrm{CD}^{+} \mathrm{T}$ cells but was generalized to $\mathrm{CD}^{+} \mathrm{T}$ cells, affecting other lymphocyte subsets such as $\mathrm{CD}^{+}$and $\gamma \delta \mathrm{T}$ cells. A potential caveat of the h-PMBC mouse model is that even with transfer of similar numbers of $\mathrm{CD}^{+} \mathrm{T}$ cells per mouse, the proportion of $\mathrm{CD}^{+} \mathrm{T}$ cells with respect to other cell subsets present in the PBMCs might be different, especially between HC and ICL donors. The presence of particular cell subsets at different proportions in the PBMC inoculum might influence the NR versus the R phenotype of the ICL patient group. Although TCR repertoire analysis or cell death measurements did not distinguish between patients in the R and NR-ICL groups, levels of Th2 cytokine production and peripheral CD8 ${ }^{+} \mathrm{T}$ cell counts were overall lower in patients with NR-ICL, suggesting the possibility of a more exhausted T cell pool. Additional work will be required to clarify these findings.

The NR-ICL group could be further divided into 2 subgroups depending on whether the inability to reconstitute the mice was the result of either a block to enter cell division or an impediment to survive under these particular in vivo conditions. We did not find a correlation between the 3 subgroups of patients with ICL and most of their immunological and clinical characteristics detailed in Table 1. Only the number of $\mathrm{CD}^{+} \mathrm{T}$ cells in blood from patients belonging to the NR-ICL group was slightly lower than in the R-ICL group. Identification of these 3 groups of patients in the ICL group could enhance further investigation of the molecular origin of lymphopenia as well as treatment strategies. In addition, it could help to identify alternative diagnoses. This was the case, for instance, of ICL-55 patient, who had Crohn's disease, a diagnosis that was confirmed several years after an "ICL" designation. The fact that he belonged to the R-ICL group in addition to the correction of his lymphopenia after anti-TNF- $\alpha$ antibody treatment, culminated with his final diagnosis of CD4 lymphopenia secondary to his Crohn's disease.

In summary, we have established that humanized mouse models with transfer of either HSCs or PBMCs from patients with ICL can be utilized to decipher ICL etiologies and pathogenesis by evaluating T cell development from HSCs as well as studying proliferation and survival of mature T cells. These models represent a real example of personalized medicine allowing us to further characterize each individual case of ICL and to assess each patient's own potential therapeutic intervention, which is especially important when dealing with such a heterogeneous syndrome of severely immunocompromised patients. 


\section{Methods}

ICL HSC and PBMC donors. Study participants provided HSCs through enrollment in the clinical study, "Hematopoietic Stem Cell Mobilization in Idiopathic CD4 Lymphocytopenia Patients and Healthy Controls for the Study of T-Cell Maturation and Trafficking in Murine Models" (P-Mobile, NCT no. 02015013) and PBMCs through enrollment in the clinical study "Etiology, Pathogenesis, and Natural History of Idiopathic CD4 ${ }^{+}$Lymphocytopenia" (EPIC, NCT no. 00867269) or through the NIH blood bank for HCs. Eligible ICL participants were adults with confirmed ICL $\left(C D 4^{+} \mathrm{T}\right.$ cell count $<300 / \mu \mathrm{L}$ at screening and on at least 2 occasions at least 6 weeks apart in the absence of any illness, treatment, or condition accounting for CD4 lymphopenia) (Table 1) and HC were eligible if healthy with a WBC count $>2,500 / \mu \mathrm{L}$ of blood and hemoglobin $\geq 12.5 \mathrm{~g} / \mathrm{dL}$. HC PBMCs were collected from healthy donors through the NIH blood bank.

CD $34^{+}$cell mobilization and purification. Study participants received $10 \mu \mathrm{g} / \mathrm{kg}$ of G-CSF (NEUPOGEN Filgastim; Amgen, Inc.) on 5 consecutive days and $0.24 \mathrm{mg} / \mathrm{kg}$ of CXCR4-blocking antibody (Plerixafor; Genzyme Corporation) on day 4. On day 5, 12 hours after Plerixafor, they underwent apheresis at the Department of Transfusion Medicine, Clinical Center, NIH, and CD34 cells were purified using antiCD34 magnetic beads (CliniMACS Plus, Miltenyi Biotec) in accordance with the procedures of the Cell Processing Section, Department of Transfusion Medicine, Clinical Center, NIH, and using a protocol approved by an NIH IRB. Purified hCD $34^{+}$cells were cryopreserved at $-140^{\circ} \mathrm{C}$ until use.

Mice. NRG and NSG mice were from The Jackson Laboratory. C57BL/6-RAGKO- $\gamma$ CKO-CD47KO, and BALB/c-RAGKO- $\gamma \mathrm{cKO}$ mice were from Taconic Farms. Both male and female mice received human cells in a blind fashion. All mice were housed in specific pathogen-free conditions. The NIH is accredited by the Association for Assessment and Accreditation of Laboratory Animal Care.

Flow cytometry. We used fluorochrome-conjugated antibodies from BD Bioscience, Biolegend, eBioscience, and Beckman Coulter as shown in Supplemental Table 1. 7-Aminoactinomycin D (7-AAD) (BD Bioscience) or LIVE/DEAD fixable Dead Cell Stain kits were used for the ultraviolet or infrared channels (Invitrogen) to exclude dead cells. Intracellular staining for Ki-67 (Biolegend) was performed using fixation/permeabilization reagents from eBioscience. Data were acquired with a Fortessa or an LSRII (both from BD Biosciences) and analyzed with FlowJo (Tree Star) software.

hCD34 mouse model. Five- or 15-week-old mice were irradiated and injected with 0.5 to 1 million CD $34^{+}$cells i.v. in $300 \mu \mathrm{L}$ of PBS within 12 hours of irradiation. They then received prophylactic antibiotics in the drinking water for 2 weeks after irradiation. Mice (6 to 12 per donor and per individual experiment) were bled and/or euthanized at different time points up to 57 weeks after CD34 $4^{+}$cell transfer. Spleen, bone marrow, and thymus were collected, desegregated in single-cell suspension, counted and stained with antibodies to analyze by flow cytometry.

hPBMC mouse model. PBMCs were thawed, counted, and stained to analyze by flow cytometry. Based on the flow data, we calculated the numbers of PBMCs (ranging from 8 to 60 million cells) required to inject per mouse in order to transfer similar numbers of $\mathrm{CD}^{+} \mathrm{T}$ and/or $\mathrm{CD}^{+} \mathrm{T}$ cells from both $\mathrm{HCs}$ and patients with ICL within the same experiment. We injected 2 to 5 mice per donor depending on the amount of PBMCs available. Mice were bled at different time points and euthanized 4 weeks after the PBMC transfer. Spleen, bone marrow, LNs, and liver were collected, and cells were counted and stained to analyze by flow cytometry. Mice were weighed at baseline and then twice a week and euthanized if they lost more than $20 \%$ of their initial body weight.

In vitro stimulation to measure cell death and cytokine production. PBMCs $\left(3 \times 10^{5}\right)$ from patients in either the HC or ICL group were cultured in duplicate 96-well U-bottom plates in the presence of just media alone or with $10^{5}$ mouse splenocytes or with plate-bound mouse anti-human CD3 antibody $(5 \mu \mathrm{g} / \mathrm{mL}$, clone OKT3, Biolegend) plus soluble mouse anti-human CD28 antibody ( $5 \mu \mathrm{g} / \mathrm{mL}$, clone CD28.2, BD Bioscience). After 24 hours, we collected the supernatants of individual wells to measure human Th cytokines (IL-5, IL-13, IL-2, IL-6, IL-9, IL-10, IFN- $\gamma$, TNF- $\alpha$, IL-17A, IL-17F, IL-4, IL-21, and IL-22) using the LEGENDplex multi-analyte flow assay kit (Biolegend) following the manufacturer's instructions. Data were acquired with a Fortessa (BD Biosciences) and analyzed with LEGENDplex data analysis software (Biolegend). The antibodies for the human cytokines used in the kit do not cross-react with mouse cytokines. Additionally, cells from duplicate wells were pooled and stained with 7-AAD and Annexin V (eBioscience) following the manufacturer's instructions. The percentage of total dead $\mathrm{CD}^{+} \mathrm{T}$ cells was calculated as the percentage of annexin $\mathrm{V}^{+}$cells within the CD3 gate (both positive and negative for 7-AAD). Percentage of apoptotic cells was calculated as the percentage of annexin $\mathrm{V}^{+} 7-\mathrm{AAD}^{-}$cells within the $\mathrm{CD} 3$ gate. The $\mathrm{CD} 3$ gate was made without a previous forward and side scatter gate to include all possible apoptotic and/or dead lymphocytes. 
Measurement of the TCR- $\beta$ repertoire. We sequenced the complementarity-determining region 3 of the TCR- $\beta$ chain from 5 donors per group (HC, NR-ICL, and R-ICL) using immunoSEQ Assay (Adaptive Biotechnologies) and analyzed the data using immunoSEQ Analyzer software (Adaptive Biotechnologies). The number of productive complementarity-determining region 3 templates analyzed per sample was at least 23,000 . Clonality represents how much of the repertoire is made up of expanded clones, and the values go from zero to one (value if all the cells in the sample belonged to a single clone).

Statistics. When showing data from individual mice, we also represent the median of the group. When representing just the median, we also show the interquartile range. When comparing 2 groups, we used the 2-tailed Mann-Whitney test and when doing multiple comparisons, we used 2-tailed ANOVA with Tukey's test correcting for multiple comparisons. A $P$ value $<0.05$ was considered significant.

Study approval. The human studies were approved by the Institutional Review Board of the National Institute of Allergy and Infectious Diseases, and informed consent was obtained from all participants prior to any study procedures in accordance with the Declaration of Helsinki. All animal experiments were approved by the Animal Care and Use Committee of the National Institute of Allergy and Infectious Diseases.

\section{Author contributions}

VS, GR, and IS recruited patients and provided patient care. APD, VS, DFS, and IS provided experimental design. APD and XL generated and analyzed experimental data with supervision from APD and IS. APD wrote the manuscript with supervision from IS.

\section{Acknowledgments}

This work was supported by the Division of Intramural Research of the National Institute of Allergy and Infectious Diseases, NIH. We thank Philip M. Murphy for critically reading the manuscript.

Address correspondence to: Ainhoa Perez-Diez, 10 Center Drive, Building 10, Room 11B17A, Bethesda, Maryland 20814, USA. Phone: 301.761.6698; Email: ainhoa@nih.gov. Or to: Irini Sereti, 10 Center Drive, Building 10, Room 11B15A, Bethesda, Maryland 20814, USA. Phone: 301.496.5533; Email: isereti@niaid.nih.gov.

VS's present address is: Center for Drug Evaluation, FDA, Silver Spring, Maryland, USA.

GR's present address is: Division of AIDS, National Institute of Allergy and Infectious Diseases, NIH, Rockville, Maryland, USA.

1. Smith DK, Neal JJ, Holmberg SD. Unexplained opportunistic infections and CD4+ T-lymphocytopenia without HIV infection. An investigation of cases in the United States. The Centers for Disease Control Idiopathic CD4 ${ }^{+}$T-lymphocytopenia Task Force. N Engl J Med. 1993;328(6):373-379.

2. Fauci AS. CD4 ${ }^{+}$T-lymphocytopenia without HIV infection—no lights, no camera, just facts. $N$ Engl J Med. 1993;328(6):429-431.

3. Zonios DI, et al. Idiopathic CD4+ lymphocytopenia: natural history and prognostic factors. Blood. 2008;112(2):287-294.

4. Mandl T, Bredberg A, Jacobsson LT, Manthorpe R, Henriksson G. CD4+ T-lymphocytopenia—a frequent finding in anti-SSA antibody seropositive patients with primary Sjögren's syndrome. J Rheumatol. 2004;31(4):726-728.

5. Baroudjian B, et al. Psoriasis associated with idiopathic CD4 ${ }^{+}$T-cell lymphopenia: a regulatory T-cell defect? Br J Dermatol. 2014;171(1):186-189.

6. Maruyama K, et al. Evaluation of cellular and humoral autoimmunity before the development of type 1 diabetes in a patient with idiopathic CD4 lymphocytopenia [published online ahead of print December 26, 2018]. J Diabetes Investig. https://doi. org/10.1111/jdi.12997.

7. Kojima M, Sakurai S, Morita Y, Nakamura N, Sugihara S, Shimano S. EBV(+) B-cell lymphoproliferative disorder associated with subsequent development of Burkitt lymphoma in a patient with idiopathic CD4(+) T-lymphocytopenia. J Clin Exp Hematop. 2008;48(2):55-59.

8. Ahmad DS, Esmadi M, Steinmann WC. Idiopathic CD4 lymphocytopenia: spectrum of opportunistic infections, malignancies, and autoimmune diseases. Avicenna J Med. 2013;3(2):37-47.

9. Menon BS, Shuaib IL, Zamari M, Haq JA, Aiyar S, Noh LM. Idiopathic CD4 ${ }^{+}$T-lymphocytopenia in a child with disseminated cryptococcosis. Ann Trop Paediatr. 1998;18(1):45-48.

10. Pasic S, Minic P, Dzudovic S, Minic A, Slavkovic B. Idiopathic CD4+ lymphocytopenia and juvenile laryngeal papillomatosis. Pediatr Pulmonol. 2005;39(3):281-283.

11. Yarmohammadi H, Cunningham-Rundles C. Idiopathic CD4 lymphocytopenia: pathogenesis, etiologies, clinical presentations and treatment strategies. Ann Allergy Asthma Immunol. 2017;119(4):374-378.

12. Régent A, et al. Idiopathic CD4 lymphocytopenia: clinical and immunologic characteristics and follow-up of 40 patients. Medi- 
cine (Baltimore). 2014;93(2):61-72.

13. Puronen CE, et al. Decreased interleukin 7 responsiveness of $\mathrm{T}$ lymphocytes in patients with idiopathic CD4 lymphopenia. $J$ Infect Dis. 2012;205(9):1382-1390

14. Hubert P, et al. [Deficiency of the CD3-TCR signal pathway in three patients with idiopathic CD $4^{+}$lymphocytopenia]. $J$ Soc Biol. 1999;193(1):11-16.

15. Bignon A, et al. DUSP4-mediated accelerated T-cell senescence in idiopathic CD4 lymphopenia. Blood. 2015;125(16):2507-2518

16. Isgrò A, Sirianni MC, Gramiccioni C, Mezzaroma I, Fantauzzi A, Aiuti F. Idiopathic CD4+ lymphocytopenia may be due to decreased bone marrow clonogenic capability. Int Arch Allergy Immunol. 2005;136(4):379-384.

17. Frühwirth M, Clodi K, Heitger A, Neu N. Lymphocyte diversity in a 9-year-old boy with idiopathic CD4 ${ }^{+}$T cell lymphocytopenia. Int Arch Allergy Immunol. 2001;125(1):80-85.

18. Laurence J, Mitra D, Steiner M, Lynch DH, Siegal FP, Staiano-Coico L. Apoptotic depletion of CD4 ${ }^{+}$T cells in idiopathic CD4+ T lymphocytopenia. J Clin Invest. 1996;97(3):672-680.

19. Scott-Algara D, et al. Idiopathic CD4+ T-cell lymphocytopenia is associated with impaired membrane expression of the chemokine receptor CXCR4. Blood. 2010;115(18):3708-3717.

20. Mosier DE, Gulizia RJ, Baird SM, Wilson DB. Transfer of a functional human immune system to mice with severe combined immunodeficiency. Nature. 1988;335(6187):256-259.

21. McCune JM, Namikawa R, Kaneshima H, Shultz LD, Lieberman M, Weissman IL. The SCID-hu mouse: murine model for the analysis of human hematolymphoid differentiation and function. Science. 1988;241(4873):1632-1639.

22. Kamel-Reid S, Dick JE. Engraftment of immune-deficient mice with human hematopoietic stem cells. Science. 1988;242(4886):1706-1709.

23. Shultz LD, et al. Multiple defects in innate and adaptive immunologic function in NOD/LtSz-scid mice. J Immunol. 1995;154(1):180-191.

24. Ito M, et al. NOD/SCID/ $\gamma(\mathrm{c})$ (null) mouse: an excellent recipient mouse model for engraftment of human cells. Blood. 2002;100(9):3175-3182.

25. Brehm MA, Jouvet N, Greiner DL, Shultz LD. Humanized mice for the study of infectious diseases. Curr Opin Immunol. $2013 ; 25(4): 428-435$.

26. Halper-Stromberg A, et al. Broadly neutralizing antibodies and viral inducers decrease rebound from HIV-1 latent reservoirs in humanized mice. Cell. 2014;158(5):989-999.

27. Aspord C, et al. Breast cancer instructs dendritic cells to prime interleukin 13-secreting CD4+ ${ }^{+}$cells that facilitate tumor development. J Exp Med. 2007;204(5):1037-1047.

28. Hogenes M, Huibers M, Kroone C, de Weger R. Humanized mouse models in transplantation research. Transplant Rev (Orlando). 2014;28(3):103-110.

29. Pagliuca FW, et al. Generation of functional human pancreatic $\beta$ cells in vitro. Cell. 2014;159(2):428-439.

30. Goettel JA, et al. Fatal autoimmunity in mice reconstituted with human hematopoietic stem cells encoding defective FOXP3. Blood. 2015;125(25):3886-3895.

31. Haddad R, et al. Molecular characterization of early human T/NK and B-lymphoid progenitor cells in umbilical cord blood. Blood. 2004;104(13):3918-3926.

32. Herndler-Brandstetter D, et al. Humanized mouse model supports development, function, and tissue residency of human natural killer cells. Proc Natl Acad Sci U S A. 2017;114(45):E9626-E9634.

33. Harris DT, Badowski M, Balamurugan A, Yang OO. Long-term human immune system reconstitution in non-obese diabetic (NOD)-Rag (-)- $\gamma$ chain (-) (NRG) mice is similar but not identical to the original stem cell donor. Clin Exp Immunol. 2013;174(3):402-413.

34. van Rijn RS, et al. A new xenograft model for graft-versus-host disease by intravenous transfer of human peripheral blood mononuclear cells in RAG2 ${ }^{-/-} \gamma \mathrm{c}^{-/-}$double-mutant mice. Blood. 2003;102(7):2522-2531.

35. Pearson T, et al. Non-obese diabetic-recombination activating gene-1 (NOD-Rag ${ }^{\text {null }}$ ) interleukin (IL)-2 receptor common gamma chain (IL2 $r^{\text {null }}$ ) null mice: a radioresistant model for human lymphohaematopoietic engraftment. Clin Exp Immunol. 2008;154(2):270-284.

36. Abele-Ohl S, Leis M, Mahmoudian S, Weyand M, Stamminger T, Ensminger SM. Rag2 $2^{-/-} \gamma$-chain ${ }^{-/}$mice as hosts for human vessel transplantation and allogeneic human leukocyte reconstitution. Transpl Immunol. 2010;23(1-2):59-64.

37. Heuts F, Gavier-Widén D, Carow B, Juarez J, Wigzell H, Rottenberg ME. CD4+ cell-dependent granuloma formation in humanized mice infected with mycobacteria. Proc Natl Acad Sci U S A. 2013;110(16):6482-6487.

38. Kovacs SB, et al. T-cell depletion in the colonic mucosa of patients with idiopathic CD4+ lymphopenia. J Infect Dis. 2015;212(10):1579-1587.

39. Signorini S, et al. Restriction of T-cell receptor repertoires in idiopathic CD4+ lymphocytopenia. Br J Haematol. 2000;110(2):434-437.

40. Kuijpers TW, et al. Idiopathic CD4+ $\mathrm{T}$ lymphopenia without autoimmunity or granulomatous disease in the slipstream of RAG mutations. Blood. 2011;117(22):5892-5896.

41. van der Burg M, et al. A DNA-PKcs mutation in a radiosensitive $\mathrm{T}^{-} \mathrm{B}^{-}$SCID patient inhibits Artemis activation and nonhomologous end-joining. J Clin Invest. 2009;119(1):91-98.

42. Elder ME, et al. Human severe combined immunodeficiency due to a defect in ZAP-70, a T cell tyrosine kinase. Science. 1994;264(5165):1596-1599.

43. Gorska MM, Alam R. A mutation in the human Uncoordinated 119 gene impairs TCR signaling and is associated with CD4 lymphopenia. Blood. 2012;119(6):1399-1406.

44. Cardinez C, et al. Gain-of-function $I K B K B$ mutation causes human combined immune deficiency. $J$ Exp Med. 2018;215(11):2715-2724.

45. Schiroli G, et al. Preclinical modeling highlights the therapeutic potential of hematopoietic stem cell gene editing for correction of SCID-X1. Sci Transl Med. 2017;9(411):eaan0820. 